\title{
Kinetics of neurodegeneration based on a risk-related biomarker in animal model of glaucoma
}

Takuya Hayashi ${ }^{1,2,3,4,5^{*}}$, Masamitsu Shimazawa ${ }^{1,6}$, Hiroshi Watabe ${ }^{1,2,7}$, Takayuki Ose ${ }^{1,2}$, Yuta Inokuchi ${ }^{6}$, Yasushi Ito ${ }^{6}$, Hajime Yamanaka', Shin-ichi Urayama ${ }^{3}$, Yasuyoshi Watanabe ${ }^{5,8}$, Hideaki Hara ${ }^{1,6}$ and Hirotaka Onoe ${ }^{1,5}$

\begin{abstract}
Background: Neurodegenerative diseases including Parkinson's and Alzheimer's diseases progress slowly and steadily over years or decades. They show significant between-subject variation in progress and clinical symptoms, which makes it difficult to predict the course of long-term disease progression with or without treatments. Recent technical advances in biomarkers have facilitated earlier, preclinical diagnoses of neurodegeneration by measuring or imaging molecules linked to pathogenesis. However, there is no established "biomarker model" by which one can quantitatively predict the progress of neurodegeneration. Here, we show predictability of a model with riskbased kinetics of neurodegeneration, whereby neurodegeneration proceeds as probabilistic events depending on the risk.
\end{abstract}

Results: We used five experimental glaucomatous animals, known for causality between the increased intraocular pressure (IOP) and neurodegeneration of visual pathways, and repeatedly measured IOP as well as white matter integrity by diffusion tensor imaging (DTI) as a biomarker of axonal degeneration. The IOP in the glaucomatous eye was significantly increased than in normal and was varied across time and animals; thus we tested whether this measurement is useful to predict kinetics of the integrity. Among four kinds of models of neurodegeneration, constant-rate, constant-risk, variable-risk and heterogeneity models, goodness of fit of the model and F-test for model selection showed that the time course of optic nerve integrity was best explained by the variable-risk model, wherein neurodegeneration kinetics is expressed in an exponential function across cumulative risk based on measured IOP. The heterogeneity model with stretched exponential decay function also fit well to the data, but without statistical superiority to the variable-risk model. The variable-risk model also predicted the number of viable axons in the optic nerve, as assessed by immunohistochemistry, which was also confirmed to be correlated with the pre-mortem integrity of the optic nerve. In addition, the variable-risk model identified the disintegrity in the higher-order visual pathways, known to underlie the transsynaptic degeneration in this disease.

Conclusions: These findings indicate that the variable-risk model, using a risk-related biomarker, could predict the spatiotemporal progression of neurodegeneration. This model, virtually equivalent to survival analysis, may allow us to estimate possible effect of neuroprotection in delaying progress of neurodegeneration.

Keywords: Diffusion tensor, Neurodegenerative mechanisms, Biomarker, Kinetic model, Glaucoma

\footnotetext{
*Correspondence: takuya.hayashi@riken.jp

${ }^{1}$ Functional Probe Research Laboratory, RIKEN Center for Molecular Imaging

Science, Kobe, Hyogo 650-0047, Japan

${ }^{2}$ Department of Investigative Radiology, National Cerebral and Cardiovascular

Center Research Institute, Suita, Osaka 565-8565, Japan

Full list of author information is available at the end of the article
}

\section{Biomed Central}

(c) 2013 Hayashi et al.; licensee BioMed Central Ltd. This is an Open Access article distributed under the terms of the Creative Commons Attribution License (http://creativecommons.org/licenses/by/2.0), which permits unrestricted use, distribution, and reproduction in any medium, provided the original work is properly cited. 


\section{Background}

Neurodegenerative diseases are slowly progressive and intractable disorders of the nervous system. The temporal profile of neurodegenerative diseases shows a pattern of high variability across patients in terms of symptoms, neuropathology and neuroimaging findings [1-3]. This variability, as well as their slowly progressive nature, poses difficulty in assessing treatment efficacy [3]. For any current and future drug that potentially delays disease progression, it is important to know whether and how much it delays deterioration caused by neurodegenerative diseases. Early detection of disease is now becoming possible by measurement of neurochemical [4,5] and neuroimaging [6] biomarkers specifically related to the pathogenic events (for review, see $[7,8]$. Although several biomarker models have been proposed to illustrate the relationship between the biomarker cascade and symptomatic/clinical stages [1,2], there is scarce evidence that biomarkers can be used to quantitatively interpret the neurodegenerative process. To this end, it is necessary to establish a model for the kinetics of neurodegeneration using a biomarker, and to predict the time courses for testing the long-term efficacy of treatment.

Classical prediction models kinetics of neurodegeneration simply in the context of accelerated aging [9], i.e., once initiated, neurodegeneration proceeds at a rate faster than in normal-aging [10] (constant-rate model in Figure 1A), but this model ignores the causality of disease-specific risk. A more elaborate interpretation has its basis in a risk-based stochastic model, in which neurodegeneration occurs as probabilistic events for each neuron depending on the risk. If the risk is time-invariable, the number of neurons should decrease as an exponential function of time, like the radioactivity decay of a radioisotope (a constant-risk model in Figure $1 \mathrm{~A})$. This was shown to be the case in vitro $[11,12]$, and in cases of hereditary neurodegeneration [11,13]. Although this model reasonably considers neurodegeneration as probabilistic events, it oversimplifies the situation of neurodegeneration in the brain. For example, the risk is not always constant in most types of non-inherited neurodegenerative diseases, as is made evident by the betweensubject variability in symptoms or neuropathologies [1-3]. In addition, interactions between neurons seem to underlie transsynaptic degeneration in the secondary neurons which have synaptic connections with primarily dying neurons [14-16]. Thus neurodegeneration is not a series of independent events like radioisotope decay. Therefore, the stochastic model needs to be qualified when neurons are exposed to a variable level of risks and/or transsynaptic death. We modeled a condition where the level of risk is time-variable (see Kinetic models of neurodegeneration in the Methods section), and thereby predicted that the number of neurons should decrease exponentially with time- integrated risk, i.e. cumulative risk (variable-risk model in Figure 1A). We also modeled transsynaptic death, the kinetics of which are linear to the primary neuronal death, thus allowing detection of the distribution of neurodegeneration across the brain.

Glaucoma, a leading cause of the adult-onset blindness, is known to involve a pathology in which axons of retinal ganglion neurons are mechanically injured by a chronic increase in intra-ocular pressure resulting from dysregulated aqueous fluid circulation [17]. In this disease, neurodegeneration occurs not only in the retinal ganglion neuronal cell bodies and their axons in the optic nerves/tracts, but also transsynaptically in the lateral geniculate nucleus, optic radiation, and visual cortex [14-16]. The causality between increased IOP and glaucomatous neurodegeneration is well established based on several observations: 1) the higher the IOP, the more accelerated is the progression of glaucoma [18]; 2) therapies that alleviate IOP prevent the progress of the disease [18]; and 3) experimental animals given a treatment that increases IOP by blocking aqueous-fluid absorption exhibit typical visual-field deficits and retinal pathologies similar to human patients $[19,20]$. Although its primary cause is different, the glaucoma shares close similarities with Parkinson's and Alzheimer's disease in several pathological findings [21] and is recently considered as one of neurodegenerative diseases that need neuroprotective therapies [22].

Here, using diffusion-weighted magnetic resonance imaging, we test whether the causal model explains the time course of neurodegeneration in the living brains of experimental animals with an established neurodegeneration model. The technique of diffusion-weighted magnetic resonance imaging has recently enabled in vivo measurements of white matter microstructure, thus allowing the quantitative, longitudinal assessment of neurodegeneration in various diseases including Alzheimer's [23,24] and Parkinson's diseases [25,26], amyotrophic lateral sclerosis [27,28] and glaucoma [29]. By applying the diffusion tensor model [30], diffusion-weighted data could be used to calculate fractional anisotropy (FA), a scale that expresses anisotropic diffusion motion and is proven to be correlated with the density of viable neuronal axons if conditions permit (for review, see [31]). We used macaque monkeys (Macaca fascicularis) with glaucoma induced by laser photocoagulation of the trabecular meshwork [32], which is known to result in a chronic increase in IOP and a pathology that mimics glaucoma, degeneration of axons of retinal ganglion neurons.

\section{Results}

The animals with glaucoma showed increased IOP in the affected eye (Figure 1B and Additional file 1: Table S1) during the follow-up period from 33 to 168 days after 

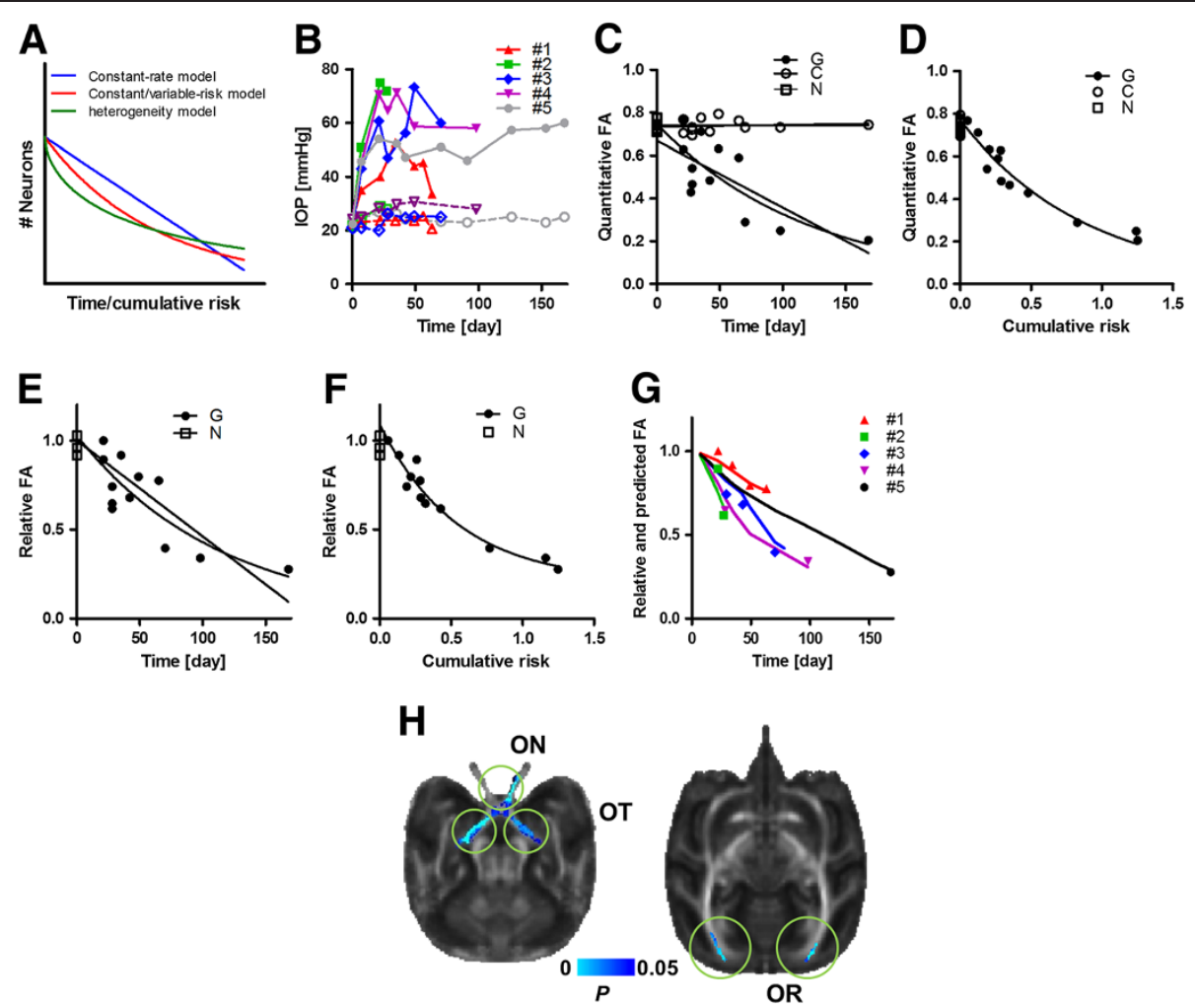

Figure 1 Kinetic model of neurodegeneration, time course of risk (intraocular pressure) and MRI-based neurodegeneration in glaucomatous animals. (A) Four putative kinetic models of neurodegeneration: 1) constant-rate model (blue straight line with $x$-axis of time after the onset of disease), 2) constant-risk model (red exponential line with $\mathrm{X}$-axis of time), 3) variable-risk model (red exponential line with $\mathrm{X}$-axis of cumulative risk), and 4) heterogeneity (+variable risk) model (green stretched exponential decay line with $x$-axis of cumulative risk). (B) Time courses of intraocular pressure (IOP) in animals with glaucoma. Closed point and thick line, the glaucomatous eye (G); open point and dashed line, control

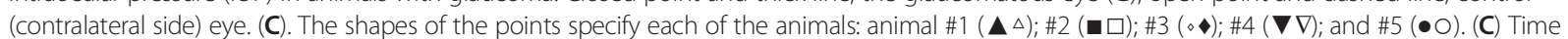
course of quantitative values of fractional anisotropy (FA) in the optic nerves of glaucomatous eye (G-ON), control (C-ON, contralateral side), and in those in normal animals (N-ON). Constant-rate model and constant-risk model are also fit to the G-ON data. See also Table 1. (D) A plot of quantitative FA values (in $y$-axis) across cumulative risk $\left(\int r(t) d t\right.$, in Eq. 2 ) in the variable-risk model (solid line). (E) Time course of relative FA values ( $r F A$ ) in the glaucomatous optic nerve expressed as a ratio to the control optic nerve $(\mathbf{G})$ and a ratio, left-to-right, in normal animals (N). Constant-rate and constant-risk models are also fit to the ratio of G. (F) A plot of rFA values in the glaucomatous optic nerve (y-axis) and cumulative risk ( $x$-axis) in glaucomatous animals $(\mathbf{G})$ and normal animals (N). The glaucomatous FA values are fit by the variable-risk model (solid line: fitted curve). (G) A plot of predicted neurodegeneration (line) and actual rFA values (points) in each animal of \#1-5. The prediction of $r F A$ were calculated based on the initial value $\left(N_{0}=1\right)$ and optimized values $\left(r_{0}=0.0019, \beta=0.057\right)$ in the variable-risk model. $(\mathbf{H})$ Results of the voxel-based analysis of FA images with cumulative IOP as a regressor. A significant cluster ( $P<0.05$, corrected for multiple comparison) is shown in blue. ON, optic nerve; OT, optic tract; and $\mathrm{OR}$, optic radiation. See Additional file 2: Table $\mathrm{S} 2$ for a list of all significant regions.

laser coagulation that blocks aqueous fluid absorption. The mean IOP values in the glaucomatous eye was higher than those in the baseline $(54.9 \pm 4.2$ vs. $23.2 \pm 0.8 \mathrm{mmHg}$, paired- $T$ test, $P<0.005)$ or than the contralateral $(22.8 \pm$ 0.73, $T$ test, $P<0.05$, Additional file 1: Table S1). In addition, as has been seen in our previous study [20], values of IOP in the glaucomatous eye were significantly variable across time (analysis of covariance, $F_{17,19}=4.34$, $P<0.05)$ and subject $\left(F_{1,19}=5.35, P<0.05\right)$ (see Figure $1 \mathrm{~B}$ ), which led us to assume that the variability in IOP fluctuates with the rate of progression of degeneration.

The FA values in the affected optic nerve were also variable across time $\left(F_{1,6}=20.0, P<0.005\right)$ and subjects $\left.\left(F_{4,6}=9.59\right), P<0.01\right)$, whereas not those of the non- affected optic nerve (Figure 1C). Besides time course of quantitative FA values, we also used those of relative FA values in the subsequent analysis of model fitting with the view to reduce effects of measurement error. The relative value of FA was expressed as a ratio of values for the affected optic nerve to those for the non-affected nerve. When we fitted the constant-rate model to the FA and time, the variance of FA was only slightly explained by time. A coefficient of determination $\left(R^{2}\right)$ was 0.35 for quantitative FA (linear regression analysis, $P<0.05$, Figure $1 C)$ and 0.36 for relative FA values $\left(P<1.0 \times 10^{-4}\right.$, Figure $1 \mathrm{E}$, see also Table 1$)$. The FA values were better fitted by the stochastic constant-risk model $\left(R^{2}=0.67\right.$, $P<1.0 \times 10^{-3}$, Figure $1 \mathrm{C}$ and $R^{2}=0.89, P<1.0 \times 10^{-5}$, 
Table 1 Parameter estimates in the constant-rate and risk-based models of primary neurodegeneration using FA values at the optic nerve

\begin{tabular}{|c|c|c|c|c|c|c|c|}
\hline & $N_{o}$ & $r_{o}$ & $\beta$ & $\gamma$ & $R^{2}$ & $P$ & $F$-test \\
\hline \multicolumn{8}{|c|}{ Constant-rate model } \\
\hline Quantitative FA & 0.64 & 0.0024 & - & - & 0.35 & $<0.05$ & - \\
\hline Relative FA & - & 0.0047 & - & - & 0.36 & $<1.0 \times 10^{-4}$ & - \\
\hline \multicolumn{8}{|l|}{ Risk-based model } \\
\hline \multicolumn{8}{|l|}{ Constant-risk model } \\
\hline Quantitative FA & 0.72 & 0.0081 & - & - & 0.67 & $<1.0 \times 10^{-3}$ & - \\
\hline Relative FA & - & 0.0084 & - & - & 0.89 & $<1.0 \times 10^{-5}$ & - \\
\hline \multicolumn{8}{|l|}{ Variable-risk model } \\
\hline Quantitative FA & 0.73 & 0.0016 & 0.064 & - & 0.94 & $<1.0 \times 10^{-6}$ & $F_{1,9}=38.6^{*}, P<0.005$ \\
\hline Relative FA & - & 0.0019 & 0.057 & - & 0.98 & $<1.0 \times 10^{-7}$ & $F_{1,10}=37.9^{*}, P<0.0005$ \\
\hline \multicolumn{8}{|l|}{ Heterogeneity model } \\
\hline Quantitative FA & 0.95 & 0.0031 & 0.060 & 0.58 & 0.96 & $<1.0 \times 10^{-7}$ & $F_{1,8}=3.5^{* *}$, N.S. \\
\hline Relative FA & - & 0.0019 & 0.057 & 0.93 & 0.98 & $<1.0 \times 10^{-8}$ & $F_{1,9}=0.6^{* *}$, N.S. \\
\hline
\end{tabular}

The quantitative and relative FA values were obtained from a region of interest in the glaucomatous optic nerve and from a ratio of FA between glaucomatous and control (contralateral) optic nerve, respectively. ${ }^{*}$ F-test compared two nested models of constant-risk and variable-risk, taking into account the additional parameter $\beta$ in the latter. ${ }^{*} F$-test compared two nested models of variable-risk and heterogeneity model, taking into account the additional parameter $\gamma$ in the latter. N.S, not statistically significant.

Figure 1D, for quantitative and relative FA data, respectively, see also Table 1). The FA data were much better fitted by the stochastic variable-risk model, based upon the cumulative risk that was assumed to be proportional to the power of the IOP (quantitative FA: $R^{2}=0.94, P<1.0 \times 10^{-6}$, Figure 1D, relative FA $R^{2}=0.98, P<1.0 \times 10^{-7}$, Figure $1 \mathrm{~F}$, Table 1). An F-test for model selection disclosed that the variable-risk model explained the data significantly better than the constant-risk (quantitative FA: $F_{1,9}=38.6$, $P<0.005$; relative FA: $F_{1,10}=37.9, P<0.0005$, Table 1 ). We also tested a model recently proposed as one that explains kinetics of neurodegeneration with heterogeneity using a stretched exponential decay function [33] (see section of Kinetic model of neurodegeneration in Methods and Figure 1A), which is often used in the field of physics to describe the relaxation in disordered system [34]. The heterogeneity model well fit to both of the quantitative and relative FA data $\left(R^{2}=0.96, P<1.0 \times 10^{-7}\right.$ and $R^{2}=0.98, P<1.0 \times 10^{-8}$, respectively, Table 1$)$, and an optimized value of parameter, $\gamma$ was less than 1 for both of quantitative and relative FA values ( 0.58 and 0.98 , respectively, Table 1) as expected in a typical stretched exponential decay model (see Methods). However, an F-test for model selection showed that the heterogeneity model did not significantly improve explanation of data variability as compared with the variable-risk model $\left(F_{1,8}=3.5 ; F_{1,9}=0.6\right.$, Table 1).

The relevant statistics were also performed with voxelbased analysis of FA images, because this might help in finding a better model if it identified significant voxels located in known pathways of degeneration, such as the optic nerve or tracts. When both the cumulative risk and the post-operative period were entered as regressors in the statistical model, the FA images revealed a cluster located in the visual pathways, including the optic nerve and tracts, and the sagittal stratum, which were significant determinants in the coefficient of cumulative risk (Figure 1E, Additional file 2: Table S2), but not in the post-operative period. In addition, the decrease in FA was specific to the laser photocoagulation treatment; such a time- or risk-dependent decrease was not found in the FA values of the contralateral optic nerve (Additional file 3: Figure S1). Because the sagittal stratum includes the optic radiation, containing axons of transsynaptic neurons, we considered that the degree of secondary neurodegeneration could be also approximated as a linear function of the degree of primary neurodegeneration (see Appendix 1).

This was tested in detail by analysis with voxel-based regression and model selection. When voxel-based multiregression analysis was performed using a regressor of the FA values at the primarily degenerated optic nerve, we found significant clusters for the contrast of primarydegeneration FA values at the bilateral optic radiation and the posterior callosum (Additional file 3: Figure S1C, Additional file 4: Table S3). These contain axons of the lateral geniculate and visual cortical neurons, respectively, which are known to be affected by transsynaptic neurodegeneration in glaucoma [16]. These results also depict the pathways to the superior colliculus, which is known to receive direct inputs from retinal neurons [35] (Additional file 3: Figure S1C, Additional file 4: Table S3). Transsynaptic neurodegeneration expressed by FA values at the optic radiation had a linear relationship with primary neuronal death as measured by FA at the optic nerve 
$\left(R^{2}=0.82, P<0.0001\right.$, Additional file 5: Table S4), suggesting that this type of pathology has its basis in tight inter-neuronal interactions for cellular survival and supporting the hypothesized protective role of neurotropic factors in transsynaptic neurodegeneration [16]. The kinetics of FA values in the transsynaptic degeneration site were also an exponential function of the cumulative risk $\left(R^{2}=0.85\right.$ in ipsilateral and $R^{2}=0.80$ in contralateral, Additional file 3: Figure S1E, Additional file 5: Table S4) and fitted by the variable-risk model significantly better than by the constant-risk model $\left(F_{1,8}=19.0, P<0.005\right.$ in ipsilateral and $F_{1,8}=14.1, \quad P<0.01$ in contralateral, Additional file 5: Table S4). Thus, these findings indicated that transsynaptic degeneration proceeds both in a linear relationship with the primary one, and in an exponential function of the cumulative risk.

Finally, we also tested whether the risk-based model could properly predict axonal degeneration. Axonal degeneration was measured by specific immunohistochemical staining with an SMI-31 antibody to evaluate phosphorylated neurofilament (NF), a marker of viable axons [36]. The NF density was better predicted by the variablerisk model than the constant-risk model by $F$-test $\left(F_{1,3}=\right.$ 29.7, $P<0.05$, Table 2 ), strengthening the validity of the current model. Because our analysis has its basis in the assumption of linearity between imaging measures of FA and biological microstructures, such as axonal densities [31], this was also tested in our test animals with their optic nerves histologically analyzed. The ratio of optic nerve FA (glaucomatous/unaffected side) was correlated with that of axonal density (Figure 2B, Spearman's $r=1.0, P<0.05$ ), which had been assessed in toluidine-blue stained sections. We also found that the FA values were significantly correlated with the density of NF-positive axons (Pearson's $r=0.92$, d.f. $=23$, one-tailed $P<0.05$, Figure $2 \mathrm{C}$, Additional file 6: Table S5).

\section{Discussion}

Using established experimental animals of neurodegeneration and in vivo neuroimaging data, we demonstrated the risk-dependent, stochastic nature of the kinetics of neurodegeneration, in which neurons probabilistically degenerate depending upon the product of time and amount of risk. Plausible exponential decay indicates that

Table 2 Parameter estimates in the risk-based kinetic models of primary neurodegeneration using the $L / R$ ratio of density of NF at the optic nerve

\begin{tabular}{lccccc}
\hline & $\boldsymbol{r}_{\boldsymbol{O}}$ & $\boldsymbol{\beta}$ & $\boldsymbol{R}^{\mathbf{2}}$ & $\boldsymbol{P}$ & F-test $^{*}$ \\
\hline Constant-risk model & 0.012 & - & 0.86 & $<0.01$ & - \\
Variable-risk model & 0.0017 & 0.071 & 0.98 & $<0.05$ & \\
& & & & & $F_{1,3}=22.4, P<0.05$
\end{tabular}

" $F$-test compared the two nested models of constant-risk and variable-risk, taking into account the additional parameter $\beta$ in the latter.

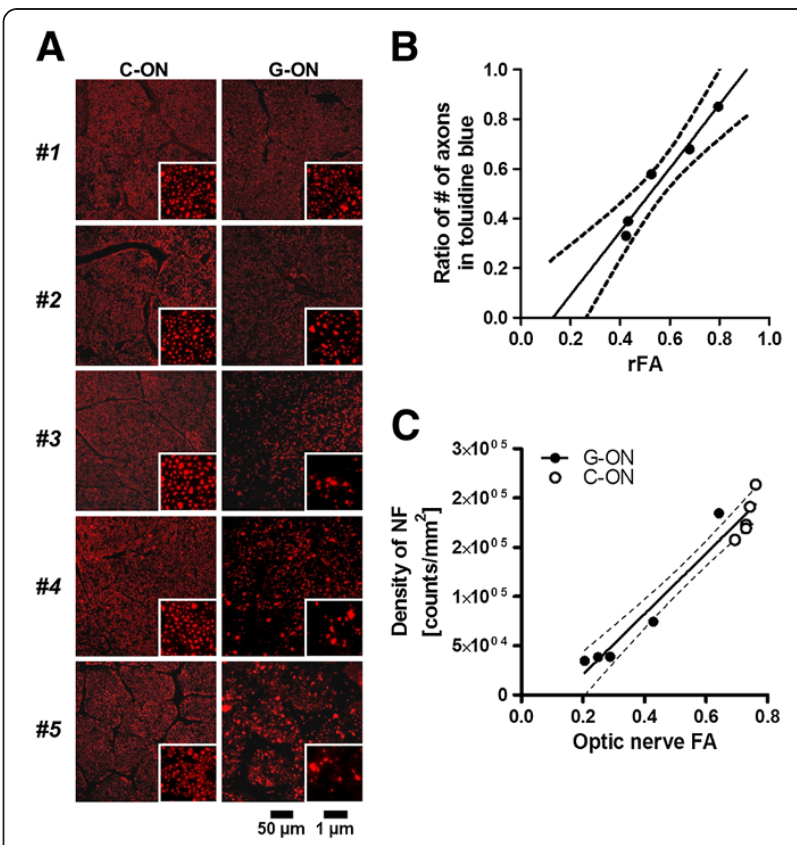

Figure 2 The relationship between a DTI-based measures, FA, and a microscopic measurements, densities of axons. (A) Sections of optic nerve immunohistochemically stained for phosphorylated neurofilaments (NF), in each side of the control (C-ON) and the glaucomatous optic nerve (G-ON), for each animal (\#1-5). The density of NF was almost comparable to that of the contralateral control in the ocular hypertensive animal (\#1), mildly decreased in the early stage glaucoma animal (\#2), and severely degraded in advanced stage animals (\#3-5). See Additional file 5: Table S5 for quantified data for each optic nerve. (B) A plot of FA values and the number of axons in the optic nerve. Values are ratios of the measured value in the glaucomatous side to that in the nonaffected side. The numbers of axons were evaluated in transaxial sections of optic nerve stained with toluidine blue. (C) The relationship between DTI-based FA values ( $x$-axis) and the microscopically-counted NF density (y-axis) in the glaucomatous (G$\mathrm{ON}$ ) and control optic nerve (C-ON). See also Additional file 5: Table S5 and Table 2.

the earlier the disease risk is accumulated, the more rapidly neurodegeneration occurs, stressing the need for earlier detection using risk-related biomarkers. The current data showed that the risk-dependent decay spatially extended not only into the optic nerve but also into the remote white matter, consistent with the known widespread pathology of glaucoma in the visual pathways including the optic nerve, lamina cribrosa, lateral geniculate nucleus, and visual cortex $[15,16]$. The IOP increase has been known not only to elicit stress on the ganglion neurons and their unmyelinated axons in the retina or/and optic head, but also to induce glial activation and expression of tumor necrosis factor $\alpha$, which may induce transsynaptic degeneration in remote areas $[16,17]$. The kinetics of the secondary transsynaptic neurodegeneration was approximated as a linear function of the kinetics of primary neurodegeneration, making the 
model effective when assessing degeneration in the brain with its enormous amount of inter-regional connectivity.

The current results provide three important findings as it relates to the kinetics of neurodegeneration. First, the results of our dynamic risk-based model for neurodegeneration confirm the stochastic nature of neurodegeneration in one-hit model, which was originally arisen from knowledge of inherited neurodegenerative diseases $[11,37]$. In the inherited trinucleotide repeat diseases, the age of symptomatic onset decreases exponentially with increasing length of trinucleotide repeats [37]. These diseases involve pathologies including initiation of nucleated polymerization, a rate-limiting thermodynamically unfavorable state, followed by rapid irreversible elongation to form fibrils $[13,38]$. Because the probability of the initial nucleation is increased by longer trinucleotide repeats, the risk function could simply be approximated as a step function across time; thus, the model explains the lengthdependent variability in the age onset $[13,37]$ and the exponential progress of neurodegeneration [11].

Second, the results extend the applicability of the riskbased stochastic model to the non-inherited, time or subject-variable neurodegenerative diseases. The variablerisk model better explained the variability of the data by $27 \%$ and $9 \%$ for quantitative and relative FA respectively $\left(R^{2}=0.94\right.$ and 0.98$)$ than did the constant-risk model $\left(R^{2}=0.67\right.$ and 0.89 , respectively). F-test for these nested models showed significant superiority of the variable-risk model (Table 1). This is important findings which indicate that the neurotoxic risk is not constant across time and subjects, even in typical animal model of neurodegeneration, which is all made by the same procedure. A majority of neurodegenerative diseases, such as Parkinson's and Alzheimer's diseases, do not involve trinucleotide repeats in the protein aggregation mechanisms and these diseases show significant between-subject variability in symptoms. This may be due to complicated pathomechanisms, such as the nonlinear nature of the risk [38,39], or multiple co-risks such as aging $[9,40]$, microcirculation [41] and microglial activation [42]. The current result showing the close relationship between pathogenic (IOP) and neurodegenerative (DTI) biomarkers is also consistent with a recent finding in Alzheimer's disease patients [43], whereby the deposition of beta-amyloid, a presumed pathogenic factor, was strongly related to hippocampal atrophy in the very early stage. Moreover, the current neurodegeneration model is virtually equivalent to those applied in the survival analysis of the Cox proportional-hazards regression model with time-dependent covariates (see Appendix 2). The model can further be generalized by including multiple risk factors (Appendix 2), such as genetic vulnerability (e.g. Apo E4 allele in Alzheimer's disease), age, sex, and microcirculation, using multiplicative combination to form the net risk function (Eq. 8). The model can also be used for estimating the effect of neuroprotection by testing the interaction between the intervention and the risk to the time course of neurodegeneration.

Third, our findings of transsynaptic neurodegeneration suggest that the regionally exposed risk can induce system-wide neurodegenerative changes. The IOP increase may only injure the ganglion neurons in the retina, but the risk-based kinetic model allowed us to detect the FA decrease in the optic radiations, the 1st order transsynaptic sites. The observations are well consistent with findings by recent studies in glaucomatous patients $[29,44]$. The effect size may not be large in the higher order transsynaptic connections (as shown in Additional file 3: Figure S1); however, a specific spatial pattern covering the primary and secondary connections may increase diagnostic specificity for neurodegeneration that often proceeds in the specific neural circuit, e.g. visual pathways in case of glaucoma.

Although the heterogeneity model with stretched exponential decay (in Eq. 6) did fit well to our data, it did not significantly better explain our data than the riskbased model. The variability of the data was largely explained by the risk-based model (94-95\%), which was improved only slightly by $1-2 \%$ when applied the heterogeneity model and F-test for model selection did not show significant difference between these two nested models (Table 1). Similarly, previous study showed that the stretched exponential model better fit to the survival curve of neurodegeneration using 16 kinds of data [33] and improved explanation of variability only by $0-11 \%$ (median $=4 \%$ ), while the (constant-) risk-based model explained $72-98 \%$ of data variability (median $=89 \%$ ). Therefore, it is possible that the heterogeneity model may better explain the neuronal survival than the riskbased model, but the effect size seems to be relatively small as compared with models with less smaller number of parameters. Moreover, we consider that the model needs to be carefully assessed for its eligibility, particularly, for the parameter, $\gamma$. The value of $\gamma$ may be influenced not only by biological factors (e.g. heterogeneity in the decay rate or late-stage biological compensatory response to the initial neurotoxic events) but also by measurement accuracy of the biomarker. Therefore, the actual factor that governs the variability of $\gamma$ should be assessed systematically in each experimental condition. As for estimation of heterogeneity of decay rates, bi-exponential decay model could be better suited than the current model to evaluate the level of heterogeneity [45]. Moreover, since adding the parameter $(\gamma)$ in the model may not only increase goodness of fit but also bias the base parameter $\left(\beta\right.$ or $\left.r_{0}\right)$, it may need to be evaluated particularly when for example the study intervenes early-stage neuroprotection that may expect any change in $\beta$. 
Based on the current results, we propose a two-step model for progression of neurodegenerative disease: a first step that correlates the "pathogenic" biomarker with the "neurodegenerative" biomarker, and a second one that correlates the neurodegenerative biomarker with clinical/symptomatic stage (Figure 3 ). This model may be similar to the recent biomarker model, where multiple biomarkers emerge in a cascade and are associated with the clinical stage [2], but it differs with respect to the emphasis on causality between cascades of biomarkers or clinical symptoms. In particular, the current risk-based stochastic kinetic model constitutes the first cascade between pathogenic and neurodegenerative biomarkers. Our approach may circumvent two problems related to biomarker accuracy. The first problem concerns the time lag between neurodegeneration and clinical manifestations. The current results show a kinetic relationship between pathogenic and neurodegenerative biomarkers, allowing prediction and therapeutic intervention to be made earlier, even in the preclinical stage. Thus, it is not necessary to do follow-up studies for a lengthy duration to await conversion from a preclinical (i.e., mild cognitive impairment, $\mathrm{MCI})$ to a clinical stage (i.e. Alzheimer's disease). In addition, inclusion of the time lag for clinical conversion may potentially have a problem of patient misclassification resulting from errors in clinical diagnosis. Modeling earlier disease stage is also important from a therapeutic point of view: the time lag between pathogenic and neurodegenerative biomarkers may be an optimal time window of neuroprotective therapies. The second problem

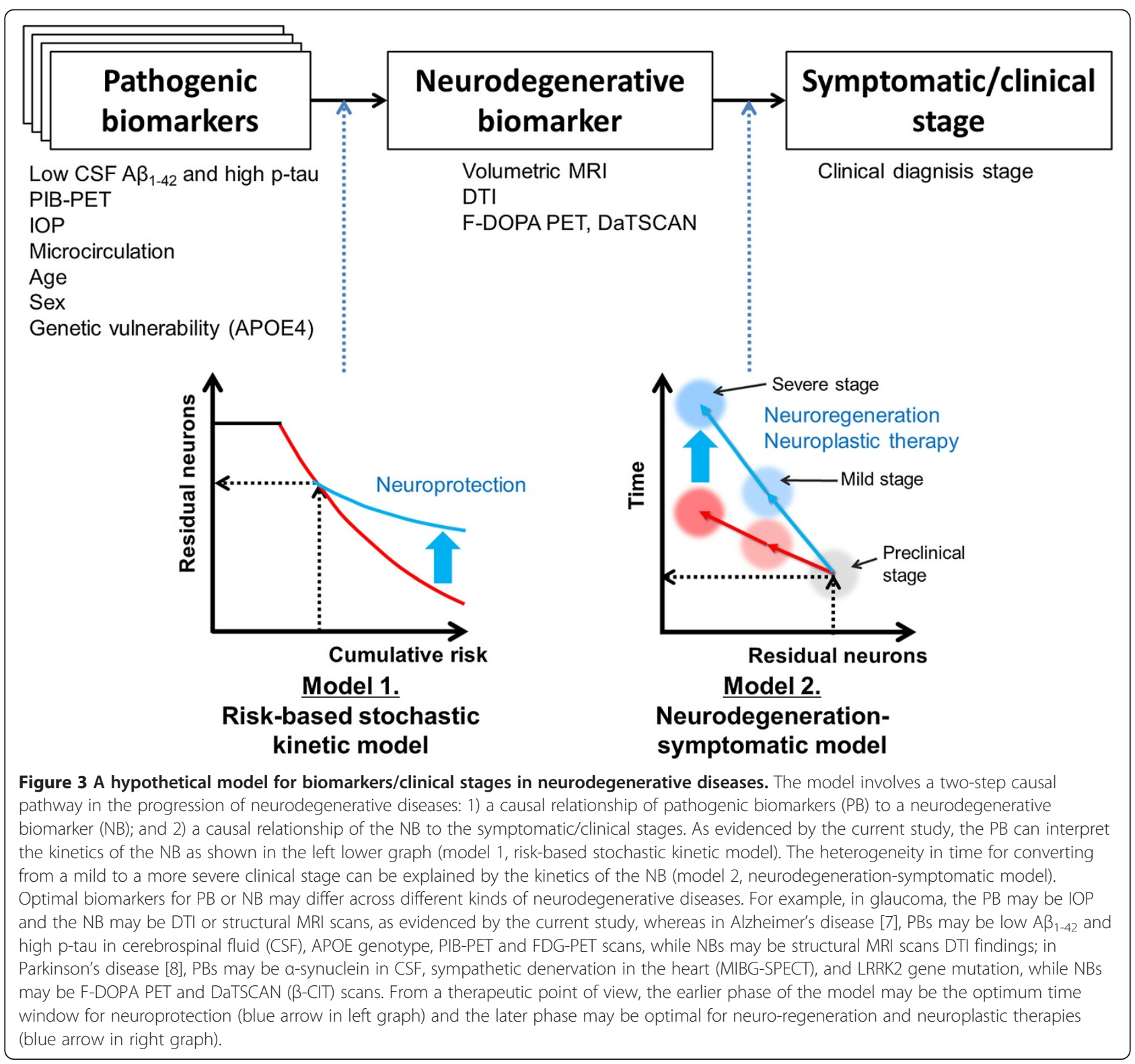


that our approach circumvents concerns degraded predictability, which is inherent when using discrete variables (clinical stages) as an outcome. Our data showed that structural MRI data, which provides quantitative values, could be used as a neurodegeneration biomarker, as evidenced by its relationship to axonal densities. Besides the early stage, our two-step biomarker model assumes later-stage causality between neurodegeneration and clinical staging (Figure 3). This is consistent with knowledge obtained from functional localization in the brain and from the lesion studies in stroke and neurodegenerative diseases; thus heterogeneity in the neurological symptoms can be explained by variable regional distribution of the neurodegeneration. Therefore, rather than the pathogenic biomarker, the neurodegeneration biomarker may be predictive for symptomatic/clinical staging. This hypothesis was supported by a recent study that showed better performance of MRI volumetry of the hippocampus (as a surrogate of neurodegeneration) than amyloid retention in the brain (used as a pathogenic biomarker) for predicting the time of conversion from MCI to AD [46]. At this later stage, therapeutic intervention may include not only neuroprotection but also neurodegenerative and neuroplastic therapies (Figure 3).

A few issues suggest the need for some caution when applying the current approach. First, to increase generalizability, the current model needs to be tested in other kinds of neurodegenerative diseases and animal models. While the origin of glaucomatous pathology is chronic physical stress to retinal ganglion cells due to continuously increased intraocular pressure, most of neurodegenerative pathologies in Parkinson' disease or Alzheimer's disease is primarily based on the biochemical events, such as abnormal protein aggregations [47]. Therefore future studies should also address potential of the current model in various pathologies of neurodegeneration. Second, the predictability of the current model may depend on the measurements of pathogenic biomarker, such as initial time point or frequency. This effect is difficult to estimate without a priori knowledge of temporal variation in the pathogenic biomarker; thus pilot studies that estimate the potential variation may be required. In terms of diseases with abnormal protein aggregation, this effect may not be large because it takes years and even decades for the accumulation of proteins to occur. Third, the predictability of the model rests on the quality of biomarker data, and this may be different across diseases. For example, in Parkinson's disease, fluorodopa PET can be used as a neurodegeneration biomarker because the uptake constant of fluorodopa has a direct linear relationship to the number of surviving dopaminergic neurons in the substantia nigra [48]. A potent pathogenic role of microglial activation can be estimated by testing the predictability of the model using
PET and ${ }^{11} \mathrm{C}-\mathrm{PK} 11195$, a marker of translocator protein $[49,50]$.

\section{Conclusions}

In summary, we have successfully explained the kinetics of glaucomatous neurodegeneration by a dynamic riskbased kinetic model and in vivo measurement of biomarkers. The results support that neurodegeneration occurs and proceeds as a probabilistic events based on the risk to neurons. Although many studies may be preferable in other kinds of neurodegenerative diseases to generalize the current kinetic model, the model may provide opportunities for predicting the time course of neurodegeneration in the living brain in the early stage of the disease, and may bridge the dissociation between heterogenous pathology in patients and standardized pathology in experimental animal models of neurodegeneration [51].

\section{Methods}

\section{Kinetic models of neurodegeneration}

In the risk-based stochastic model, the rate of decrease in neuronal/axonal density is a product of the risk of cell death at time $t, r(t)$, and the density of neurons/axons, $N(t)$, which can be expressed as follows,

$$
d N(t) / d t=-r(t) \cdot N(t)
$$

The $r(t)$ may be thought of as the instantaneous probability of cell death at a particular time, $t$. Solving differential equations of Eq. 1 generates the following equation:

$$
N(t)=N_{0} \exp \left(-\int r(t) d t\right)
$$

where $N_{O}$ is the number of neurons at time zero. The function for risk, $r(t)$, should be non-negative and could be substituted by an exponential function as follows:

time-variable risk:

$$
r(t)=r_{0} \cdot \exp (\beta \cdot p(t))
$$

or

time-invariable (constant) risk:

$$
r(t)=r_{0}
$$

where $r_{O}$ constitutes a component of baseline risk, while the other term, $\exp (\beta \cdot p(t))$, constitutes the risk. In the variable-risk model (Eq. 3), the risk for glaucoma, intraocular pressure (IOP), was incorporated into the $p(t)$, which was calculated as measured IOP values minus $29 \mathrm{mmHg}$, the $95 \%$ confidence upper limit of IOP in the normal optic nerve. The optimal values for $r_{O}, \beta, N_{O}$ were estimated to obtain the best fit of the model by a non- 
linear least square method using the values of IOP and FA in the glaucomatous optic nerves at each time point.

For the constant-risk model, $\beta$ in Eq. 3 was fixed to zero, thus forming Eq. 4, which corresponds to that used in previous literature (Eq. 3 in ref. [11]), and the values of $r_{O}$ and $N_{O}$ were optimized in the fitting procedure. Besides these two risk-based models, we also evaluated the classical model (constant-rate model) of neurodegeneration [10], in which neuronal loss proceeds at a constant rate, as follows:

$$
d N(t) / d t=-r_{0}
$$

where $r_{O}$ is a rate constant for the decrease in the number of neurons, expressed as counts per unit of time. The initial condition was again substituted by $N(0)=N_{0}$.

In addition, we considered a similar but another model of neurodegeneration kinetics, recently proposed for heterogeneous kinetics [33]. The model has been often used in the field of physics or engineer to describe the discharge of capacitors, relaxation of disordered systems or luminescence decay [34], and is recently applied to several biological data such as estimation of human lifespan [52] and kinetics of heterogeneous neurodegeneration [33].

$$
N(t)=N_{0} \exp \left(-\left(\int r(t) d t\right)^{\gamma}\right)
$$

In this model, $\gamma$ is a shape parameter such that if $0<\gamma$ $<1, N(t)$ shows a typical form of stretched exponential decay across $\int r(t) d t$, while if $\gamma=1$ it becomes identical to the original risk-based exponential decay as in Eq. 2, and if $\gamma>1$ sigmoidal (or compressed exponential) decay. Mathematically, the function is also known as a Weibull function [53].

\section{Animals}

Eight macaque monkeys (Macaca fascicularis, body weight $4-5 \mathrm{~kg}$, all male) were used; five animals were used to model glaucomatous pathologies, while three were control. The animal model of glaucoma was made using an established method of laser photocoagulation, as described previously [20]. Glaucoma model animals were evaluated in detail for histological changes in microglial cells, astrocytes, and neurons in the lateral geniculate nucleus, and the results were published elsewhere [50]. Visual field defects and pathological features of this animal model are also described in detail in our previous studies $[19,20,54]$. Before being enrolled into these experiments, all animals were confirmed by ophthalmoscopy to have no abnormalities in their ocular fundus. After glaucoma was induced, the animals were repeatedly followed up with measurements of intraocular pressure (IOP) and diffusion tensor imaging. All surgical and experimental procedures conformed to $\mathrm{NIH}$ guidelines for the care of experimental animals (National Institutes of Health Committee on Care and Use of Laboratory Animals, 1985). The study was approved by the Institutional Animal Care and Use Committee of RIKEN and the National Cardiovascular Center Research Institute.

\section{Induction of experimental glaucoma}

Elevated IOP was induced by applying argon blue/green laser photocoagulation burns to the trabecular meshwork of the left eye, with the right eye being used as an untreated control. For the laser treatment, the animals were anesthetized with an intramuscular injection of ketamine $(8.75 \mathrm{mg} / \mathrm{kg})$ plus xylazine $(0.5 \mathrm{mg} / \mathrm{kg})$. A single-mirror Goldmann lens filled with a physiological solution was placed on the eye to be treated. The argon laser was focused on the mid-portion of the trabecular meshwork, and a total of 150 laser-beam spots were applied around $360^{\circ}$ (spot size $100 \mu \mathrm{m}$; power $1.0 \mathrm{~W}$; exposure time $0.2 \mathrm{sec}$ ) using an argon laser photocoagulator (Ultima 2000 SE; Coherent Inc., CA, USA) attached to a standard slit-lamp microscope (BQ 900; Haag-Streit, Köniz, Switzerland). The same laser treatment was repeatedly applied two weeks after the first treatment to maintain continuous IOP elevation, as has been confirmed previously. Potential complications of the glaucoma surgery, including retinal ischemia were not suspected by detailed histological evaluations [50].

\section{IOP}

The IOP was intermittently measured (over an interval of 3-28 days) in both eyes during the course of glaucoma in each animal using a calibrated applanation pneumotonometer (Model 30 Classic Pneumotonometer; Medtronic Solan, FL, USA). The measurement was performed under generalized anesthesia using intramuscular ketamine $(8.75-10 \mathrm{mg} / \mathrm{kg}$ ) and local anesthesia with $0.4 \%$ oxybuprocaine hydrochloride.

\section{DTI}

Diffusion tensor magnetic resonance images (DTI) were acquired using a 3-Tesla magnetic resonance image (MRI) scanner (Signa Horizon Lx VH3, General Electric Healthcare, Little Chalfont, Buckinghamshire, UK), which provides a maximum gradient strength of $40 \mathrm{mT}$ $\mathrm{m}^{-1}$, rising in $268 \mu \mathrm{s}$ using a customized eight-channel phased array receiver coil. During the DTI scanning, animals were intubated and deeply anesthetized using a gaseous anesthetic, $1.5 \%$ isoflurane, with their respiration assisted by a ventilator (Cato, Dräger, Germany). To maintain stable physiological conditions, we monitored the partial pressures of oxygen, carbon dioxide and isoflurane in the inspiratory and expiratory gases. We also intermittently performed gas analysis of the arterial blood 
that was collected from the tail artery, in which a 24gauge needle was indwelled. The animal's head was fixated to a customized acrylic retainer that was firmly attached to the receiver coil. The animal and the retainer were placed on the MRI gantry with the animal's head centered in the bore of MRI scanner. After scanning a localizer for planning a field of view for subsequent scans, a higherorder shimming was performed using a spiral sequence that allows us to calculate the fitted field map including second-order components (for a total of 10 components), and to minimize inhomogeneity in the static magnetic field. Then, the DTI data were collected using a multishot spin-echo type EPI sequence (number of shot $=2$, $\mathrm{TR}=17000 \mathrm{~ms}, \mathrm{TE}=81.9 \mathrm{~ms}, \mathrm{FA}=90^{\circ}$, number of slice $=51)$ with an isotropic spatial resolution of $0.9 \mathrm{~mm}$ and with diffusion-weighted gradients of 27 directions ( $\mathrm{b}$ value $\left.=1000 \mathrm{~s} \mathrm{~mm}^{-2}\right)$. The diffusion-weighted gradients were applied in a symmetrical, twice-refocused pulse to reduce eddy-current-induced distortion in the DTI images [55]. The DTI data, a total of 24 volumes for three nondiffusion-weighted volumes and 21 diffusion-weighted volumes, were obtained by three separate scans, each consisting of one non-diffusion and seven diffusionweighted volumes. Before each scan, we monitored the temperature of the gradient coil, and started the scan when the temperature was between $22^{\circ}$ and $23^{\circ} \mathrm{C}$, at which the shift and distortion across scans were minimized. If the temperature was less than that specified, we performed dummy scans to keep the temperature within range. The DTI data were scanned three times for each time point during the follow-up of glaucoma. The total time for obtaining DTI data was approximately 5 hours, depending on the temperature and computation time for the image reconstruction. We also scanned two gradientecho sequences with different $\mathrm{TE}$ values $(\mathrm{TR}=200 \mathrm{~ms}$, $\mathrm{TE} 1=4.4 \mathrm{~ms}$, TE2 $=6.637 \mathrm{~ms}$ ) and obtained the field map to be used for a post-process of reducing distortion of DTI images, which originates from the magnetic field inhomogeneities caused by magnetic susceptibility differences between neighboring tissues, such as air-bone or air-tissue.

\section{Image analysis}

Raw DTI data were corrected for image shift, rotation and distortion (with a linear transformation of 12 degrees of freedom) and for B0-inhomogeneity distortion (with nonlinear warping calculated on the fieldmap) using the programs FLIRT and PRELUDE, respectively, both part of the Functional magnetic resonance imaging of the brain (FMRIB) Software Library (FSL) [56], developed by the Analysis Group of FMRIB Centre, University of Oxford. Estimated shifts for these corrections were concurrently applied to the original images to minimize reslicing noise in the corrected data. Then, the image for fractional anisotropy (FA) was computed by fitting a diffusion tensor model [30] to the corrected diffusion data using the program, FDT, part of the FSL.

For region of interest (ROI) analysis of FA based on the primary degeneration kinetic model, we delineated the ROI with a columnar shape ( $3 \mathrm{~mm}$ long, diameter of $2 \mathrm{~mm}$, number of voxels of 12), centered at the center of the optic nerve, at a distance of $6 \mathrm{~mm}$ from the junction of the eyeball and the nerve. For each ROI, we obtained FA values averaged across three scans obtained at the same time point. For fitting the kinetic models of primary neurodegeneration, we used a quantitative value of FA or ratio of FA ( $r F A$, expressed as a ratio to the FA of the contralateral optic nerve) in glaucomatous animals and determined optimum values for $N_{O}, r_{O}$ and $\beta$. When we used $r F A, N_{O}$ was fixed to one. The integration of risk function, $r(t)$, in Eq. 1 was calculated based on the trapezoid rule using discrete time point data for IOP. The goodness of fit was evaluated based on coefficients of determination $\left(R^{2}\right)$ and the $P$-value computed in the fitting process. The $F$-test for model selection was performed for the two nested models between with constant- and variable-risk or between with variable-risk and heterogeneity. The fitting and $F$-test were computed using Pybld (http://www.mi.med.osaka-u.ac.jp/pybld/pybld. html) built in the language Python. We also confirmed that the goodness of fits in the stochastic variable-risk model was independent of the arbitrarily determined threshold (29 mmHg, 95\% confidence upper limit of normal IOP). For presenting graphs, the optimized values determined at the fitting process for FA data, was used for $N_{0}, r_{0}$ and $\beta$ to calculate the cumulative risk, $\int \exp (r(t)) d t$ to be used in the $\mathrm{x}$-axis of graphs (Figure 1D, 1F and Additional file 3: Figure $\mathrm{S} 1 \mathrm{E})$ and for making the regressor in the subsequent analysis of voxel-based statistics and of secondary neurodegeneration (see below). FA and rFA values in normal animals were used for the scatter plot, but not for fitting the kinetic models of neurodegeneration. For graph plotting, the cumulative risks of control (contralateral to glaucomatous side) and normal optic nerves were considered to be zero, which means that baseline risk, $r_{0}$, was also zero.

For voxel-based analysis of FA images, we used tractbased spatial statistics (TBSS) [57], part of the FSL. First, FA images were brain-extracted using BET [58] and were aligned into a common space (a matrix of $137 \times$ $167 \times 100$; a voxel size of $0.4 \mathrm{~mm}$-cubic) using the nonlinear registration tool FNIRT, part of the FSL, which uses a b-spline representation of the registration warp field [59], followed by linear registration to the standard space of the anterior-posterior commissure line of the macaque brain [60]. Next, the mean FA image was created and thinned to create a mean FA skeleton representing the centers of all tracts common to all data. The mean FA skeleton was created by thresholding the 
mean FA image at an FA value larger than 0.3. Each scan's aligned FA data were then projected onto this skeleton and the resulting data fed into voxel-wise crosssubjects statistics for the linear regression analysis. The statistical analysis for estimation of the neurodegenerative model included analyzing the skeletonized FA images by testing significance of coefficients of post-operative time and the cumulative risk in the multi-regression statistical model. The analysis for the secondary neurodegenerative changes involved analyzing the skeletonized FA images by testing regressors of FA values in the affected optic nerve and of post-operative time. The predictor in the variablerisk model was made by using the optimized values for $N_{O}, r_{O}$ and $\beta$ determined from prior analysis of the quantitative FA in the primary degenerated area, the optic nerve (Table 1). For the statistical threshold, we applied threshold-free cluster-corrected P-values less than 0.05 . Voxel-wise statistics were performed by permutationbased nonparametric inference using the program Randomise, part of the FSL. For post hoc analysis of the model of secondary neurodegeneration, a cubic ROI consisting of 27 voxels was placed with the center of the ROI located at the local maximum in the optic radiation in the contrast for the regressor of optic nerve FA. The model equation, Eq. 6, was fitted to the FA values in the areas of primary degeneration (glaucomatous optic nerve) and secondary degeneration (optic radiation), and the optimum values for $k_{t}$ and $N_{B O}$ were determined. The model was also built based on the risk, by substituting $N_{A}(t)$ in Eq. 6 by Eq. 2, then was fitted to the FA data of the optic radiation to determine optimum values for $N_{O}, r_{0}$, and $N_{B O}$. An $F$-test was performed for model selection between constant- and variable-risk models, taking into account the additional parameter $(\beta)$ in the latter.

\section{Histology}

The animals were analyzed by conventional histological evaluations, including toluidine blue and cresyl violet staining of retina, optic nerve and lateral geniculate nucleus. In addition, to correlate the optic nerve DTI changes with the degeneration of axons, optic nerve immunofluorescence staining was performed using SMI-31 antibody, a maker of neurofilament (NF), a constituent of neuronal axons. Animals were euthanized 33-168 days after glaucoma induction under deep general anesthesia (isoflurane in $\mathrm{N}_{2} \mathrm{O}$ and $\mathrm{O}_{2}$ by inhalation) and were perfused via the common carotid artery with $1 \mathrm{~L}$ of $0.9 \%$ saline containing $10 \mathrm{U} / \mathrm{ml}$ heparin at room temperature, followed by $1 \mathrm{~L}$ of $4 \%$ paraformaldehyde in $0.01 \mathrm{M}$ phosphate-buffered saline (PBS; pH 7.4). Eyeballs with optic nerves were enucleated at the time of brain removal and 4\% paraformaldehyde in PBS solution was injected into the vitreous body and postfixed by immersion in $4 \%$ paraformaldehyde in PBS for at least 1 week at $4^{\circ} \mathrm{C}$. After immersion, a 3-mm long segment of optic nerve was cut out at 6 to $9 \mathrm{~mm}$ from the eyeballoptic nerve junction. The eyeballs and optic nerve segments were soaked in 10,15 , and $30 \%(\mathrm{w} / \mathrm{v})$ sucrose in $0.1 \mathrm{M}$ phosphate buffer, $\mathrm{pH} 7.4$, at $4^{\circ} \mathrm{C}$, for at least 24 $\mathrm{h}$ each, and then frozen in embedding compound (Tissue-Tek; Sakura Finetechnical Co.Ltd., Tokyo, Japan).

Coronal sections of the optic nerve segment were cut at $10-\mu \mathrm{m}$ thickness and every 20th section was mounted onto the same slide glass until we obtained 20 slide glasses each containing four sections. Because cutting of the sections started from the end closer to the eyeball, the sections evaluated for counting should be 6-7 mm distant from the eyeball-nerve junction. Optic nerve sections were washed with $0.01 \mathrm{M}$ PBS, preincubated with $10 \%$ normal goat serum in $0.01 \mathrm{M}$ PBS for $30 \mathrm{~min}$, and then incubated overnight at $4^{\circ} \mathrm{C}$ with mouse anti-SMI31 monoclonal antibody (NE1022; 1: 1000, Calbiochem, San Diego, CA, USA). They were washed with $0.01 \mathrm{M}$ PBS and then incubated for $3 \mathrm{~h}$ at room temperature with Alexa Fluor $546 \mathrm{~F}\left(\mathrm{ab}^{\prime}\right) 2$ fragment of goat anti-mouse IgG ( $\mathrm{H}+\mathrm{L})$ (1:1000 dilution) (Molecular Probes, Eugene, OR). For counting the number of SMI-31-positive axons, we randomly chose a single slide glass from the twenty, and identified five regions (nasal, temporal, superior, inferior, and central) in each of the four optic nerve sections. In the center of each region, we placed a sample volume of interest with a size of $218 \times 164 \times 10 \mu \mathrm{m}$ (in $\mathrm{x}, \mathrm{y}$, and section thickness) under a microscope fitted with a $40 \times$ objective $\times 3$ digital zoom; thus, a total of $0.18 \mathrm{~mm}^{2}$ per section (areal ratio of $3.6 \pm 0.4 \%$ as mean \pm S.E.) was assessed for counting the number of SMI-31-positive (i.e. NF-positive) axons. Counting and photography were performed by a single observer who was blinded to the animals' data and DTI results (Y. I). Thereby, for each optic nerve, we obtained 20 measurements of axon densities (five regions $\times$ four sections) and calculated the mean value that represents the NF-positive axon density of the corresponding optic nerve.

The obtained NF-positive axon density was used as an independent variable in the linear regression analysis of optic nerve FA. The ratio of NF density (between the glaucomatous and contralateral optic nerve) was used for fitting the risk-based kinetic models of neurodegeneration and determining the optimum values for $r_{O}$, and $\beta$. Model selection was also analyzed using an $F$-test between constant- and variable-risk models. Although the absolute NF density in monkey \#1 (mild stage glaucomatous animal) was almost comparable with those in normal nerves (Additional file 6: Table S5), the ratio (to that in the contralateral non-affected optic nerve) was slightly decreased (0.86), similar to the axonal density (0.85) as evaluated by toluidine blue staining. In fact, toluidine blue-stained 
sections of this animal's affected optic nerve revealed neurodegeneration and formation of myelin ovoids in the peripheral part of the optic nerve.

\section{Appendix 1}

Model for secondary (transsynaptic) neurodegeneration We considered a case in which region A suffers from primary neurodegeneration due to primary neurotoxic risk, while a remote area, region $\mathrm{B}$, which is connected to region A, suffers from the risk of transneuronal neurodegeneration due to the primary degeneration in region $\mathrm{A}$. The kinetics of secondary neurodegeneration in region $\mathrm{B}\left(N_{B}(t)\right)$ was assumed to have a linear relationship with that of the primary neurodegeneration in region $\mathrm{A}\left(N_{A}(t)\right)$ as follows,

$$
\frac{d N_{B}(t)}{d t}=k_{t} \cdot \frac{d N_{A}(t)}{d t}
$$

where $k_{t}$ is a constant coefficient. The value of $k_{t}$ should depend both on the strength of the trans-synaptic neurodegeneration and the connectivity between regions $\mathrm{A}$ and $\mathrm{B}$, which may vary from region to region, or connectivity strength to the primary lesion in the brain. The differential equation (Eq. 7) indicates that the number of neurons in the area $\mathrm{B}\left(D_{B}(t)\right)$ should decrease as a linear function of the primary degenerated area $\mathrm{A}\left(D_{A}(t)\right)$ (the intercept of this linear function was substituted by $N_{B O}$ ) as illustrated in Additional file 3: Figure S1A. Thus, if the neurodegeneration kinetics of the latter region is an exponential function of the cumulative risk (Eq. 2), the kinetics in region $\mathrm{B}$ should also be a decremental exponential function across the cumulative risk, but approach the plateau, $N_{B O}$, at infinite time (Additional file 3: Figure S1B).

\section{Appendix 2}

\section{General risk-based kinetic model of neurodegeneration}

As an extension of the time-variable risk model, we also considered the situation where multiple factors are involved in the risk of neurodegeneration. If these multiple risk factors expose one to neurodegenerative risk independently, the net risk is supposed to be a multiplication of the risk function of each risk factor. Thus, the net risk function in Eq. 3 can be generalized into the following equation:

$$
r(t)=r_{0} \cdot \exp \left(\beta_{1} \cdot p_{1}(t)+\beta_{2} \cdot p_{2}(t)+\cdots+\beta_{n} \cdot p_{n}(t)\right)
$$

The general form of neurodegeneration kinetics, as formulated in Eq. 2 and 7, is virtually equivalent to those used in the survival analysis of the Cox hazard proportional regression model with time-dependent covariates $[61,62]$. Thus, the treatment effect could be estimated by testing the significance of a covariate for the treatment or an interaction term between the pathogenic biomarker and the treatment.

\section{Additional files}

Additional file 1: Table S1. List of animals with monocular

glaucomatous neurodegeneration.

Additional file 2: Table S2. Significant FA changes and their correlation with cumulative risk.

Additional file 3: Figure S1. Transsynaptic secondary

neurodegeneration - kinetic model and findings in glaucomatous animals.

Additional file 4: Table S3. Significant FA changes and their correlation with FA values for the glaucomatous optic nerve.

Additional file 5: Table S4. Parameter estimates in the kinetic models of secondary neurodegeneration using FA values at the optic radiation (OR).

Additional file 6: Table S5. Quantitative results of immunostained phosphorylated neurofilaments (NF) in the optic nerve in monocular glaucomatous model animals.

\section{Competing interests}

There are no non-financial competing interests to declare in relation to this manuscript.

\section{Authors' contributions}

TH carried out the MRI experiments, analysis and wrote the manuscript. MS carried out the preparation of animal model and helped to draft the manuscript. HW wrote the program for kinetic model. TO carried out the MRI experiment. YI carried out IOP measurements. HY carried out MRI experiment. SU wrote the MRI sequence program. HW participated in the design of the study. $\mathrm{HH}$ participated in the design of the stuty. $\mathrm{HO}$ participated in the design of the study and coordination. All authors read and approved the final manuscript.

\section{Acknowledgments}

We thank Akihide Yamamoto and Hiroshi Sato for their technical help, Kyoko Shioya for her generous support for handling animals, and Keizo Sugaya for his careful review of the manuscript. This study was supported by a Grant for Molecular Imaging Science Program from Ministry of Education, Culture, Sports, Science and Technology (MEXT), Japan; a KAKENHI Grant from MEXT [21613011 to T.H.], a Health Labour Sciences Research Grant from The Ministry of Health Labour and Welfare, Japan [H17-kokoro-025 to T.H.], and a Grant from the National Institute of Biomedical Innovation [10-05 to T.H.].

\section{Author details}

${ }^{1}$ Functional Probe Research Laboratory, RIKEN Center for Molecular Imaging Science, Kobe, Hyogo 650-0047, Japan. ²Department of Investigative Radiology, National Cerebral and Cardiovascular Center Research Institute, Suita, Osaka 565-8565, Japan. ${ }^{3}$ Human Brain Research Center, Kyoto University Graduate School of Medicine, Kyoto 606-8507, Japan. ${ }^{4}$ Center for iPS cell Research and Application, Kyoto University, Kyoto 606-8507, Japan. ${ }^{5}$ Department of Physiology, Osaka City University Graduate School of Medicine, Osaka 545-8586, Japan. ${ }^{6}$ Molecular Pharmacology, Department of Biofunctional Evaluation, Gifu Pharmaceutical University, Gifu 501-1196, Japan. ${ }^{7}$ Faculty of Molecular Imaging in Medicine, Osaka University Graduate School of Medicine, Osaka 565-0871, Japan. ${ }^{8}$ Molecular Probe Dynamics Laboratory, RIKEN Center for Molecular Imaging Science, Kobe, Hyogo 650-0047, Japan.

Received: 18 May 2012 Accepted: 4 January 2013

Published: 18 January 2013

\section{References}

1. DeKosky ST, Marek K: Looking backward to move forward: early detection of neurodegenerative disorders. Science 2003, 302:830-834. 
2. Jack CR Jr, Knopman DS, Jagust WJ, Shaw LM, Aisen PS, Weiner MW, Petersen RC, Trojanowski JQ: Hypothetical model of dynamic biomarkers of the Alzheimer's pathological cascade. Lancet Neurol 2010, 9:119-128.

3. Komarova NL, Thalhauser CJ: High Degree of Heterogeneity in Alzheimer's Disease Progression Patterns. PLoS Comput Biol 2011, 7:e1002251.

4. Fjell AM, Walhovd KB, Fennema-Notestine C, McEvoy LK, Hagler DJ, Holland D, Brewer JB, Dale AM: CSF biomarkers in prediction of cerebral and clinical change in mild cognitive impairment and Alzheimer's disease. J Neurosci 2010, 30:2088-2101.

5. Perneczky R, Tsolakidou A, Arnold A, Diehl-Schmid J, Grimmer T, Förstl H, Kurz A, Alexopoulos P: CSF soluble amyloid precursor proteins in the diagnosis of incipient Alzheimer disease. Neurology 2011, 77:35-38.

6. Dickerson BC, Wolk DA: MRI cortical thickness biomarker predicts AD-like CSF and cognitive decline in normal adults. Neurology 2012, 78:84-90.

7. Shaw LM, Korecka M, Clark CM, Lee VM-Y, Trojanowski JQ: Biomarkers of neurodegeneration for diagnosis and monitoring therapeutics. Nat Rev Drug Discov 2007, 6:295-303.

8. Sherer TB: Biomarkers for parkinson's disease. Sci Transl Med 2011, 3:79ps14.

9. Drachman DA: Aging of the brain, entropy, and Alzheimer disease. Neurology 2006, 67:1340-1352.

10. Dunnett SB, Bjorklund A: Prospects for new restorative and neuroprotective treatments in Parkinson's disease. Nature 1999, 399:A32-A39.

11. Clarke G, Collins RA, Leavitt BR, Andrews DF, Hayden MR, Lumsden CJ, McInnes RR: A one-hit model of cell death in inherited neuronal degenerations. Nature 2000, 406:195-199.

12. Dubinsky JM, Kristal BS, Elizondo-Fournier M: On the probabilistic nature of excitotoxic neuronal death in hippocampal neurons. Neuropharmacology 1995, 34:701-711.

13. Sugaya K, Matsubara S: Nucleation of protein aggregation kinetics as a basis for genotype-phenotype correlations in polyglutamine diseases. Mol Neurodegener 2009, 4:29.

14. Clark LG: The laminar organization and cell content of the lateral geniculate body in the monkey. J Anat Lond 1941, 75:419-433.

15. Yoles E, Schwartz M: Degeneration of Spared Axons Following Partial White Matter Lesion: Implications for Optic Nerve Neuropathies. Exp Neurol 1998, 153:1-7.

16. Yucel Y, Gupta N: Glaucoma of the brain: a disease model for the study of transsynaptic neural degeneration. Prog Brain Res 2008, 173:465-478.

17. Kwon $\mathrm{YH}$, Fingert $\mathrm{JH}$, Kuehn MH, Alward WL: Primary open-angle glaucoma. N Engl J Med 2009, 360:1113-1124.

18. Leskea MC, Heijl A, Hyman L, Bengtsson B, Komaroff E: Factors for progression and glaucoma treatment: the Early Manifest Glaucoma Trial. Curr Opin Ophthalmol 2004, 15:102-106.

19. Sasaoka M, Nakamura K, Shimazawa M, Ito Y, Araie M, Hara H: Changes in visual fields and lateral geniculate nucleus in monkey laser-induced high intraocular pressure model. Exp Eye Res 2008, 86:770-782.

20. Shimazawa M, Tomita G, Taniguchi T, Sasaoka M, Hara H, Kitazawa Y, Araie M: Morphometric evaluation of changes with time in optic disc structure and thickness of retinal nerve fibre layer in chronic ocular hypertensive monkeys. Exp Eye Res 2006, 82:427-440.

21. Gupta N, Yücel YH: Glaucoma as a neurodegenerative disease. Curr Opin Ophthalmol 2007, 18:110-114.

22. Danesh-Meyer HV: Neuroprotection in glaucoma: recent and future directions. Curr Opin Ophthalmol 2011, 22:78-86.

23. Douaud G, Jbabdi S, Behrens TEJ, Menke RA, Gass A, Monsch AU, Rao A, Whitcher B, Kindlmann G, Matthews PM, Smith S: DTI measures in crossing-fibre areas: increased diffusion anisotropy reveals early white matter alteration in $\mathrm{MCl}$ and mild Alzheimer's disease. Neuroimage 2011 55:880-890.

24. Rose SE, Chen F, Chalk JB, Zelaya FO, Strugnell WE, Benson M, Semple J, Doddrell DM: Loss of connectivity in Alzheimer's disease: an evaluation of white matter tract integrity with colour coded MR diffusion tensor imaging. J Neurol Neurosurg Psychiatr 2000, 69:528-530.

25. Yoshikawa K, Nakata Y, Yamada K, Nakagawa M: Early pathological changes in the parkinsonian brain demonstrated by diffusion tensor MRI. J Neurol Neurosurg Psychiatr 2004, 75:481-484.

26. Péran $P$, Cherubini $A$, Assogna F, Piras F, Quattrocchi $C$, Peppe $A$, Celsis $P$, Rascol O, Démonet J-F, Stefani A, Pierantozzi M, Pontieri FE, Caltagirone C, Spalletta G, Sabatini U: Magnetic resonance imaging markers of Parkinson's disease nigrostriatal signature. Brain 2010, 133:3423-3433.
27. Ellis CM, Simmons A, Jones DK, Bland J, Dawson JM, Horsfield MA, Williams SC, Leigh PN: Diffusion tensor MRI assesses corticospinal tract damage in ALS. Neurology 1999, 53:1051-1058.

28. Sach M, Winkler G, Glauche V, Liepert J, Heimbach B, Koch MA, Büchel C, Weiller C: Diffusion tensor MRI of early upper motor neuron involvement in amyotrophic lateral sclerosis. Brain 2004, 127:340-350.

29. Garaci FG, Bolacchi F, Cerulli A, Melis M, Spanò A, Cedrone C, Floris R, Simonetti $G$, Nucci $C$ : Optic nerve and optic radiation neurodegeneration in patients with glaucoma: in vivo analysis with 3-T diffusion-tensor MR imaging. Radiology 2009, 252:496-501

30. Basser PJ, Mattiello J, LeBihan D: MR diffusion tensor spectroscopy and imaging. Biophys J 1994, 66:259-267.

31. Beaulieu C: Diffusion MRI. In From Quantitative Measurement to In Vivo Neuroanatomy. Edited by Johansen-Berg H, Behrens T. London: Elsevier; 2009.

32. Quigley HA, Hohman RM: Laser energy levels for trabecular meshwork damage in the primate eye. Invest Ophthalmol Vis Sci 1983, 24:1305-1307.

33. Clarke G, Lumsden CJ: Scale-free neurodegeneration: cellular heterogeneity and the stretched exponential kinetics of cell death. J Theor Biol 2005, 233:515-525.

34. Huber DL: Statistical model for stretched exponential relaxation in macroscopic systems. Phys Rev B 1985, 31:6070-6071.

35. Kondo $Y$, Takada M, Tokuno $H$, Mizuno N: Single retinal ganglion cells projecting bilaterally to the lateral geniculate nuclei or superior colliculi by way of axon collaterals in the cat. J Comp Neurol 1994, 346:119-126.

36. Poltorak M, Freed WJ: Immunoreactive phosphorylated epitopes on neurofilaments in neuronal perikarya may be obscured by tissue preprocessing. Brain Res 1989, 480:349-354.

37. Gusella JF, MacDonald ME: Molecular genetics: unmasking polyglutamine triggers in neurodegenerative disease. Nat Rev Neurosci 2000, 1:109-115.

38. Murphy RM: Kinetics of amyloid formation and membrane interaction with amyloidogenic proteins. Biochim Biophys Acta 2007, 1768:1923-1934

39. Ashe KH, Zahs KR: Probing the biology of Alzheimer's disease in mice. Neuron 2010, 66:631-645

40. Douglas PM, Dillin A: Protein homeostasis and aging in neurodegeneration. J Cell Biol 2010, 190:719-729.

41. Craft S: The role of metabolic disorders in Alzheimer disease and vascular dementia: two roads converged. Arch Neurol 2009, 66:300-305.

42. Prinz M, Priller J, Sisodia SS, Ransohoff RM: Heterogeneity of CNS myeloid cells and their roles in neurodegeneration. Nat Neurosci 2011, 14:1227-1235.

43. Chételat $G$, Villemagne $V L$, Bourgeat P, Pike KE, Jones $G$, Ames D, Ellis KA Szoeke C, Martins RN, O'Keefe GJ, Salvado O, Masters CL, Rowe CC Relationship between atrophy and beta-amyloid deposition in Alzheimer disease. Ann Neurol 2010, 67:317-324

44. El-Rafei A, Engelhorn T, Wärntges S, Dörfler A, Hornegger J, Michelson G: A framework for voxel-based morphometric analysis of the optic radiation using diffusion tensor imaging in glaucoma. Magn Reson Imaging 2011, 29:1076-1087.

45. Assaf $Y$, Cohen $Y$ : Non-mono-exponential attenuation of water and Nacetyl aspartate signals due to diffusion in brain tissue. J Magn Reson 1998, 131:69-85.

46. Jack CR Jr, Wiste HJ, Vemuri P, Weigand SD, Senjem ML, Zeng G, Bernstein MA, Gunter JL, Pankratz VS, Aisen PS, Weiner MW, Petersen RC, Shaw LM, Trojanowski JQ, Knopman DS: Brain beta-amyloid measures and magnetic resonance imaging atrophy both predict time-to-progression from mild cognitive impairment to Alzheimer's disease. Brain 2010, 133:3336-3348.

47. Brundin P, Melki R, Kopito R: Prion-like transmission of protein aggregates in neurodegenerative diseases. Nat Rev Mol Cell Biol 2010, 11:301-307.

48. Pate BD, Kawamata T, Yamada T, McGeer EG, Hewitt KA, Snow BJ, Ruth TJ, Calne DB: Correlation of striatal fluorodopa uptake in the MPTP monkey with dopaminergic indices. Ann Neurol 1993, 34:331-338.

49. Gerhard A, Pavese N, Hotton G, Turkheimer F, Es M, Hammers A, Eggert K, Oertel W, Banati RB, Brooks DJ: In vivo imaging of microglial activation with [11C](R)-PK11195 PET in idiopathic Parkinson's disease. Neurobiol Dis 2006, 21:404-412.

50. Shimazawa M, Ito $Y$, Inokuchi $Y$, Yamanaka H, Nakanishi T, Hayashi T, Ji B, Higuchi M, Suhara T, Imamura K, Araie M, Watanabe Y, Onoe H, Hara H: An alteration in the lateral geniculate nucleus of experimental glaucoma monkeys: In vivo positron emission tomography imaging of glial activation. PLoS One 2012, 7:e30526

51. Obeso JA: Modeling clinical features of neurodegeneration. Nat Med 2010, 16:1372. 
52. Weon BM, Je JH: Theoretical estimation of maximum human lifespan. Biogerontology 2009, 10:65-71.

53. Weibull W: A statistical distribution function of wide applicability. J App/ Mech 1951, 18:293-297.

54. Ito $\mathrm{Y}$, Shimazawa M, Chen $\mathrm{Y}-\mathrm{N}$, Tsuruma K, Yamashima T, Araie M, Hara H: Morphological changes in the visual pathway induced by experimental glaucoma in Japanese monkeys. Exp Eye Res 2009, 89:246-255.

55. Reese TG, Heid O, Weisskoff RM, Wedeen VJ: Reduction of eddy-current -induced distortion in diffusion MRI using a twice-refocused spin echo. Magn Reson Med 2003, 49:177-182.

56. Smith SM, Jenkinson M, Woolrich MW, Beckmann CF, Behrens TE, JohansenBerg H, Bannister PR, De Luca M, Drobnjak I, Flitney DE, Niazy RK, Saunders J, Vickers J, Zhang Y, De Stefano N, Brady JM, Matthews PM: Advances in functional and structural MR image analysis and implementation as FSL. Neuroimage 2004, 23(Suppl 1):S208-S219.

57. Smith SM, Jenkinson M, Johansen-Berg H, Rueckert D, Nichols TE, Mackay CE, Watkins KE, Ciccarelli O, Cader MZ, Matthews PM, Behrens TE: Tract-based spatial statistics: voxelwise analysis of multi-subject diffusion data. Neuroimage 2006, 31:1487-1505.

58. Smith SM: Fast robust automated brain extraction. Hum Brain Mapp 2002, 17:143-155.

59. Rueckert D, Sonoda LI, Hayes C, Hill DL, Leach MO, Hawkes DJ: Nonrigid registration using free-form deformations: application to breast MR images. IEEE Trans Med Imaging 1999, 18:712-721.

60. Bowden DM, Martin MF: Primate Brain Maps: Structure of the Macaque Brain: A Laboratory Guide with Original Brain Sections, Printed Atlas and Electronic Templates for Data and Schematics. 1st edition. Amsterdam: Elsevier Science; 2000.

61. Cox DR: Regression Models and Life-Tables. J Statist Assoc Series B (Methodological) 1972, 34:187-220.

62. Fisher LD, Lin DY: Time-dependent covariates in the Cox proportionalhazards regression model. Annu Rev Public Health 1999, 20:145-157.

doi:10.1186/1750-1326-8-4

Cite this article as: Hayashi et al: Kinetics of neurodegeneration based on a risk-related biomarker in animal model of glaucoma. Molecular Neurodegeneration 2013 8:4.

\section{Submit your next manuscript to BioMed Central and take full advantage of:}

- Convenient online submission

- Thorough peer review

- No space constraints or color figure charges

- Immediate publication on acceptance

- Inclusion in PubMed, CAS, Scopus and Google Scholar

- Research which is freely available for redistribution 